\title{
ELECTRONIC STRUCTURE OF ZINC-BLENDE AND HEXAGONAL SEMICONDUCTORS: COMPARATIVE THEORETICAL STUDY*
}

\author{
J. KONIOR \\ Institute of Physics, Jagiellonian University, Reymonta 4, 30-059 Kraków, Poland \\ AND S. KAPRZYK \\ Academy of Mining and Metallurgy, Institute of Physics and Nuclear Technique \\ Al. A. Mickiewicza 30, 30-059 Kraków, Poland
}

\begin{abstract}
Using a self-consistent Korringa-Kohn-Rostoker Green function method and the local density approximation for the exchange and correlation potentials, we calculated the electronic structure and the corresponding densities of states for two semiconducting compounds that exist in both cubic (zinc-blende) and hexagonal (wurtzite and NiAs-type) crystal structures, i.e., for CdSe and MnTe. Due to the use of the same method of calculation for both structures a comparison between the obtained results and finding the factors that determine the difference between the properties of these two phases are possible. The presented local density approximation-KorringaKohn-Rostoker results might be of importance for the understanding of the factors that determine the relative stability of different phases of semiconducting compounds.
\end{abstract}

PACS numbers: $71.10 .+x$

\section{Introduction}

Cubic and hexagonal semiconductors are the most common structures in which the binary semiconductors crystallize [1]. Moreover, there are some compounds that exist in both of these two structures. From the cubic structures, the zinc-blende (zb) is the most common, while the wurtzite (w) and the NiAs-type (hex) structures should be mentioned as those originating from the hexagonal root. There are numerous papers in which the electronic, optical, and structural properties of cubic or hexagonal compounds have been investigated, both experimentally and theoretically (see, for example, Ref. [2] and references therein). However, to

*This work was partially supported by the State Committee for Scientific Research (Republic of Poland), grant number PB 938/2/91. 
our knowledge, there were no study where the electronic properties of the same compound would have been examined in both structures by the same theoretical method. In this paper we present such a theoretical comparison using an $a b$ initio Green function Korringa-Kohn-Roster (KKR) method [3] based on the local density approximation (LDA) for the correlation and exchange potentials. As the model examples, we chose CdSe (in $\mathrm{zb}$ and w structures) and MnTe (in $\mathrm{zb}$ and hex structures); for these compounds we use the notation $\mathrm{CdSe}(\mathrm{w}), \mathrm{CdSe}(\mathrm{zb})$, $\mathrm{MnTe}(\mathrm{hex})$, and $\mathrm{MnTe}(\mathrm{zb})$, respectively. The paper is organized as follows. In Sec. 2, we describe our KKR electronic structure calculations. Section 3 presents the obtained densities of states and some other numerical data. Finally, Sec. 4 gives the conclusions that follow from the calculations.

\section{KKR electronic structure calculations}

Using the self-consistent KKR method, we performed the $a b$ initio band structure calculations for $\mathrm{CdSe}(\mathbf{w})$ and $\mathrm{CdSe}(\mathbf{z b})$, and for $\mathrm{MnTe}(\mathrm{hex})$ and $\mathrm{MnTe}(\mathbf{z b})$ compounds. We took the experimental values of the lattice constants $[1,4,5]$; they are listed in Table. These data and the ion types placed in an elementary cell of

TABLE

Assumed lattice constants used in the KKR calculations and some obtained data. Here $E_{d}$ is the position of the $d$ states as measured with respect to the Fermi energy and $E_{\mathrm{g}}$ is the value of the fundamental energy gap. The experimental values, when available, are given in the text.

\begin{tabular}{l|c|c|c|c}
\hline \hline & \multicolumn{2}{|c|}{ CdSe } & \multicolumn{2}{c}{ Mn'Te } \\
\cline { 2 - 5 } & zinc-blende & wurtzite & zinc-blende & (hex) NiAs-type \\
\hline Lattice & $a=6.050$ & $a=4.299$ & $a=6.338$ & $a=4.132$ \\
constants $[\AA]$ & & $c=7.010$ & & $c=6.712$ \\
\hline Magnetic & & & & \\
moments $\left[\mu_{\mathrm{B}}\right]$ & - & - & $4.186(\mathrm{Mn})$ & $3.884(\mathrm{Mn})$ \\
$E_{d}[\mathrm{eV}]$ & 8.799 & 8.399 & - & - \\
$E_{\mathrm{g}}[\mathrm{eV}]$ & 1.565 & 1.495 & 0 & 0
\end{tabular}

the crystal were the only input data in our computational scheme. With these, the one-particle Schrödinger equation was solved with the assumption that there is a muffin-tin (MT) sphere around each ion such that the effective one-particle crystal potential may be assumed as being spherically symmetric inside the MT spheres. The muffin-tin spheres around the ionic positions were chosen to have a radius equal to half of the distance between the nearest-neighbor ions. There were also additional "empty" MT spheres introduced in order to fill the empty space between the ionic MT spheres. To calculate the wave function, the spherical function expansion was used; the expansion was truncated at $l_{\max }=2\left(l_{\max }=1\right)$ 
for the "ionic" ("empty") MT spheres. The core electrons were treated in a full relativistic way, while for the valence electrons a semirelativistic approach was applied. The self-consistency criterion was that the difference between the input and output potential should be smaller than $0.001 \mathrm{Ry}$. As the output we got the projected densities of states (DOS) and the band structure of a given compound. Some of these results are presented in the next subsection.

\section{Numerical results}

\section{1. $C d S e$}

For CdSe, the wurtzite structure is believed to be a stable one. We performed the KKR calculations for $\mathrm{CdSe}(w)$ and Fig. 1 presents the total DOS resulting from our calculations. The same figure presents also total DOS as calculated for

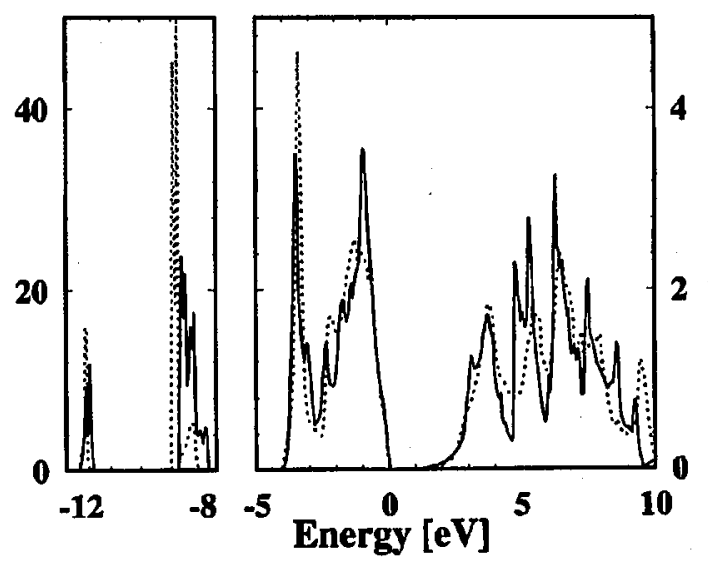

Fig. 1. Variation of the total density of states (DOS) for CdSe as calculated by the KKR method; solid line is for $\mathrm{CdSe}(\mathbf{w})$ and dotted line is for $\mathrm{CdSe}(\mathbf{z b})$ structure. Zero of the energy scale is chosen at the Fermi level $E_{\mathrm{F}}$. Note the different energy scales for two parts of the figure.

$\mathrm{CdSe}(\mathrm{zb})$. Generally, these two curves are similar in shape, though there are some differences that should be mentioned. First, the $\operatorname{CdSe}(\mathbf{z b}) s$ - and $d$-bands, placed around 12 and $8.5 \mathrm{eV}$ below the Fermi energy $E_{\mathrm{F}}$, respectively, are shifted toward lower energies when compared with the same bands of $\mathrm{CdSe}(\mathrm{w})$. The position of the $d$-bands is usually denoted as $E_{d}$ and its calculated values are given in Table. For $\mathrm{CdSe}(\mathrm{w})$, we got $E_{d}=-8.399 \mathrm{eV}$ and this result is in better agreement with the experimental value $E_{d}=-10.04 \mathrm{eV}[6]$ than the value of $E_{d}=-7.18 \mathrm{eV}$ obtained earlier with the pseudopotential method [7]. Second, for $\mathrm{CdSe}(\mathrm{zb})$ the highest peak in the valence band is observed near the bottom of the band, while in $\mathrm{CdSe}(\mathrm{w})$ the spectral weight of DOS is shifted to the top of the valence band. This difference might be of importance, for example, for transport properties, where DOS near 
the Fermi level plays an important role. Third, the $\mathrm{CdSe}(\mathrm{zb})$ conduction band has more pronounced peaks and they should manifest in the X-ray absorption spectra, like X-ray absorption near-edge structure (XANES) [8]. Finally, we have calculated values of the fundamental energy gap $E_{\mathrm{g}}$ and they are given in Table. As usually, the LDA results underestimate the experimental value of $E_{\mathrm{g}}$; for $\mathrm{CdSe}($ w) $E_{\mathrm{g}}=1.84 \mathrm{eV}[1]$.

\section{2. $M n T e$}

In normal conditions, MnTe has the NiAs-type crystalline structure. Further, the magnetic moments of Mn atoms are ordered antiferromagnetically (AF) along the $c$-axis, while in the $a-b$ plane the same moments are ordered ferromagnetically. We performed the KKR band structure calculations for such an MnTe crystal and the obtained projected Mn-atom densities of states are presented in Fig. 2. Because of space limitation, we decided to present only these Mn-atom

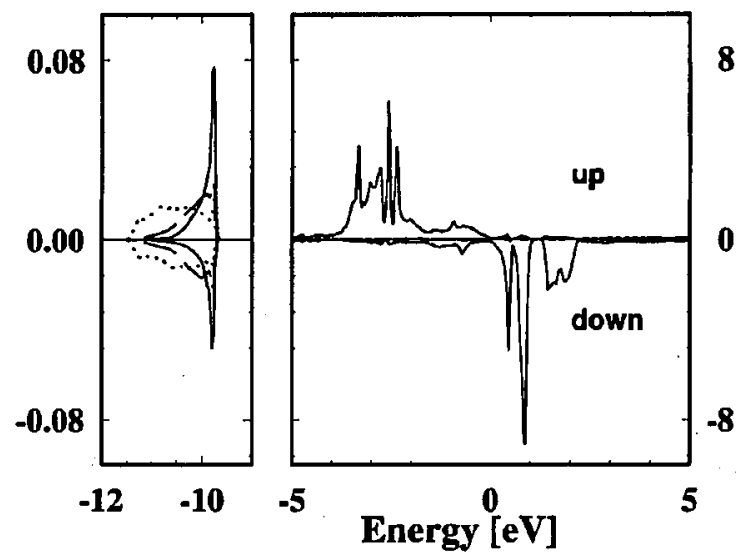

Fig. 2. Variation of the projected Mn-atom density of states (DOS) for MnTe(hex) as calculated by the KKR method; dotted, dashed, and solid line are for the $s, p$, and $d$ type DOS, respectively. Zero of the energy scale is chosen at the Fermi level $E_{\mathrm{F}}$. Note the different energy scales for two parts of the figure.

densities of states because they entirely determine the magnetic properties of the compound. The obtained magnetic moment of both AF sublattices is given in Table and it is lower than the corresponding experimental value equal to $\approx 5.1 \mu_{\mathrm{B}}$ [9]. The $\mathrm{MnTe}(\mathrm{zb})$ crystals have been recently obtained by the molecular beam epitaxy (MBE) method [5] and so far no information is available about its magnetic structure. The importance of the $\operatorname{MnTe}(\mathrm{zb})$ phase follows also from the fact that this compound is another extreme limit of the so-called "semimagnetic semiconductors", like $\mathrm{Cd}_{1-x} \mathrm{Mn}_{x} \mathrm{Te}$, for example [10]. We performed our KKR calculations for the $\mathrm{MnTe}(\mathrm{zb})$ with the ferromagnetic ordering of $\mathrm{Mn}$ spins. The calculated projected Mn-atom DOS is presented in Fig. 3; the corresponding magnetic moment of the Mn ions is given in Table and we have no data to confront this result 


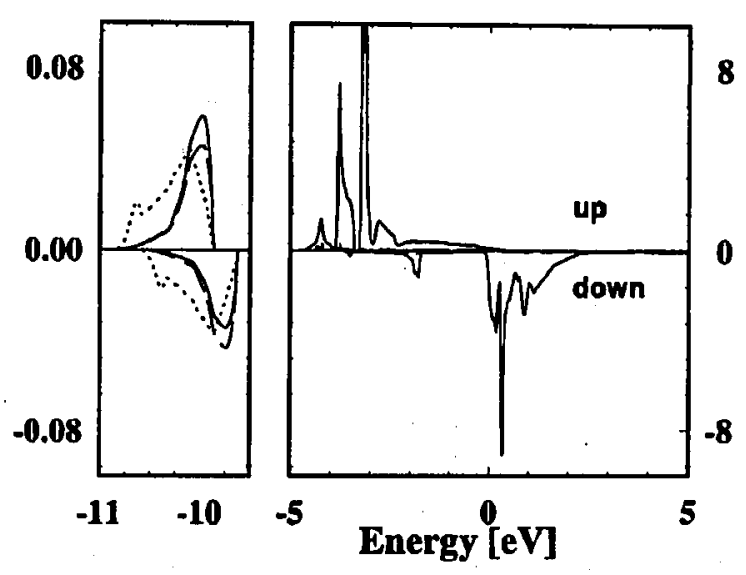

Fig. 3. Variation of the projected Mn-atom density of states (DOS) for MnTe(zb) as calculated by the KKR method; dotted, dashed, and solid line are for the $s, p$, and $d$ type DOS, respectively. Zero of the energy scale is chosen at the Fermi level $E_{\mathrm{F}}$. Note the different energy scales for two parts of the figure.

with the experiment. A direct comparison between the two figures, i.e., Figs. 2 and 3 , is difficult because of different assumed magnetic structure of $\mathrm{MnTe}(\mathrm{hex})$ and $\mathrm{MnTe}(\mathrm{zb})$. Nevertheless, some remarks can be done. First, in both compounds there is no energy gap at the Fermi level, though close to the Fermi $E_{\mathrm{F}}$ energy the values of DOS are small. In both structures, the Mn $d$-type states dominate close to $E_{\mathrm{F}}$. Next in magnitude Te $p$-states represent around $50 \%$ and $10 \%$ for $\mathrm{MnTe}($ hex) and $\mathrm{MnTe}(\mathrm{zb})$, respectively. Third, for lower bands (around $10 \mathrm{eV}$ below the Fermi level) the values of DOS are small and all three components (of $s$, $p$, and $d$ type) are comparable in magnitude.

\section{Conclusions}

We performed the $a b$ initio self-consistent calculations of CdSe and $\mathrm{MnTe}$ compounds, each in two crystalline structures. We have discussed the difference in electronic structure which is produced by different lattice symmetry. The next step of our calculation should also include the optimizing geometry and that would allow us to connect the changes in the electronic structure with the stability of different crystalline phases.

\section{Acknowledgments}

The authors thank Prof. J. Kossut for stimulating discussions. 


\section{References}

[1] M.L. Cohen, J.R. Chelikowsky, Electronic Structure and Optical Properties of Semiconductors, Springer-Verlag, New York 1988.

[2] Ch.-Y. Yeh, Z.W. Lu, S. Fröyen, A. Zunger, Phys. Rev. B 46, 10086 (1992).

[3] J. Korringa, Physica 13, 392 (1946); W. Kohn, N. Rostoker, Phys. Rev. 94, 1111 (1954); S. Kaprzyk, preprint, Academy of Mining and Metallurgy, Kraków 1982.

[4] J. Soltys, private communication.

[5] J. Kossut, private communication.

[6] L. Ley, R.A. Pollack, F.R. McFeely, S.P. Kowalczyk, D.A. Shirley, Phys. Rev. B 9, 600 (1974).

[7] P. Schröer, P. Krüger, J. Pollmann, Phys. Rev. B 48, 18264 (1993).

[8] J. Konior, A. Kisiel, J. Oleszkiewicz, S. Kaprzyk, E. Burattini, A. Mycielski, submitted for publication.

[9] E. Uchida, H. Kondoh, N. Fukuoka, J. Phys. Soc. Jpn. 11, 27 (1956).

[10] Semiconductors and Semimetals, Eds. J.K. Furdyna, J. Kossut, Vol. 25, Academic, New York 1988. 